\title{
Mauricio o las elecciones primaria d'Eduardo Mendoza : des personnages et une ville en proie au desencanto
}

Marie-Thérèse Figueroa

\section{(2) OpenEdition}

Journals

Édition électronique

URL : https://journals.openedition.org/cher/3855

DOI : $10.4000 /$ cher.3855

ISSN : 2803-5992

Éditeur

Presses universitaires de Strasbourg

\section{Édition imprimée}

Date de publication : 1 décembre 2015

Pagination : 197-206

ISBN : 978-2-86820-913-9

ISSN : 1968-035X

Référence électronique

Marie-Thérèse Figueroa, « Mauricio o las elecciones primaria d'Eduardo Mendoza : des personnages et une ville en proie au desencanto », reCHERches [En ligne], 15 | 2015, mis en ligne le 01 décembre 2021, consulté le 15 décembre 2021. URL : http://journals.openedition.org/cher/3855 ; DOI : https:// doi.org/10.4000/cher.3855

\section{c) (i) (2)}

Ce(tte) œuvre est mise à disposition selon les termes de la Licence Creative Commons Attribution Pas d'Utilisation Commerciale - Partage dans les Mêmes Conditions 4.0 International. 


\title{
Mauricio o las elecciones primaria d'Eduardo Mendoza: des personnages et une ville en proie au desencanto ${ }^{1}$
}

\author{
MARIE-THÉRÈSE FIGUEROA \\ Aix Marseille Université, CAER
}

\section{L’Espagne postfranquiste: une société en pleine mutation}

Après l'épisode traumatique de la Guerre civile, la vie politique espagnole est cadenassée par le général Franco, vainqueur du conflit. Or rien ne laissait envisager que les institutions mises en place devaient permettre, le moment venu, une amorce d'ouverture vers la démocratie. À la mort de Franco, le roi Juan Carlos I accède au pouvoir comme l'avait prévu le dictateur. Rien ne doit bouleverser l'ordre établi depuis la fin de la Guerre civile : « todo está atado y bien atado » selon la formule consacrée. Et pourtant l'Espagne va vivre une succession de bouleversements, de "crises» sur le plan politique, économique et sociétal. Les premières élections démocratiques de juin 1977, la création des Autonomies, la reconnaissance du Parti Communiste Espagnol, la Constitution, les accords de la Moncloa, le 23-F, l'affaiblissement de l'Église catholique donnent lieu à une véritable "crise» de l'identité nationale. Dans ce contexte, un mouvement tel que La Movida va se construire aux antipodes de certaines valeurs dont celle de la famille traditionnelle. Cette nouvelle société, née à partir des structures mises en place dans les années 1960, repose en partie sur l'évolution de la mentalité des forces d'opposition, sur les mutations sociologiques fruit du fort développement économique, sur des sentiments nationalistes périphériques sans cesse affirmés, sur le nouveau positionnement de l'Église de plus en plus

1 Ce mot apparaît dans la sphère publique dès le début des années 1980. Jaime Chávarri, réalisateur du film El desencanto (1976) utilise déjà ce vocable comme titre de son film qui narre la saga de la famille Panero.

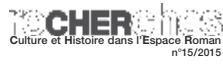


critique envers l'État franquiste, sur la faiblesse croissante du Movimiento qui se désagrège inexorablement, ou encore sur la nécessité urgente de rejoindre la Communauté européenne. Dans ce délicat passage vers la démocratie la mémoire se met en veille dès les premières années afin de préserver les chances de réussite d'un processus qui sera salué internationalement. Eduardo Mendoza, fer de lance d'une génération d'écrivains d'après guerre, situe son roman Mauricio o las elecciones primarias dans les années quatre-vingt, à la veille des élections autonomiques en Catalogne. Il met en scène trois personnages et leurs acolytes qui, par leurs réflexions, leur analyse de la société catalane, dépeignent non seulement la situation politique, sociale et économique de cette autonomie particulière mais aussi les transformations qu'a vécues l'Espagne durant le lent processus de transition qui mène le pays d'une dictature à une démocratie moderne. Barcelone, ville phare de ce roman, est le centre d'une action complexe où l'heure des bilans est arrivée. Le desencanto semble régner dans toutes les sphères de la société comme réponse aux désillusions nées du changement impossible: "de lo que pudo haber sido y no fue». Mais, est-ce une simple posture intellectuelle des personnages? La ville en est-elle le reflet? N'assistonsnous pas à l'éclosion d'une nouvelle société pragmatique où le paraître semble de mise, où la fracture sociale s'agrandit, où seule la perspective des futurs Jeux olympiques semble apaiser les dissensions politiques et sociales... ? Ne sommesnous pas en présence d'une jeunesse qui aurait abandonné le train de l'illusion collective?

Mendoza nous propose une vision polyphonique de l'Espagne postfranquiste et de la Catalogne en particulier, mais son roman ne se limite pas à une étude des mécanismes sociaux et politiques qui ont produit le desencanto. Il est indéniable toutefois que la politique y occupe une place importante, sans que le lecteur y prenne toujours garde. La tonalité nous est donnée dès le départ. Les anges déchus ainsi que les géants - ils ouvrent et ferment le roman, leur disparition est regrettée par quelques inconditionnels - sont la métaphore de la chute des grandes idéologies et du grand rêve de liberté sexuelle, comme l'explicite l'auteur dans un entretien publié dans El País semanal'. L'objectif de ce travail est d'analyser la manière dont s'inscrit le desencanto, d'une part, dans la caractérisation des

2 Mendoza E., El País semanal, 26/03/2006: «Dudé mucho en poner ese inicio y ese final. Todo se sustentaba sobre una capa de hielo tan fina que en cierto modo necesitaba metaforizar, darle cierto espesor ejemplificador a la historia. Los gigantes son las grandes ideologías caídas, y sólo un grupo de nostálgicos parece echarlas en falta $[\ldots]$.

-El sida es desde luego el gran asunto de la década de los ochenta. Usted recrea perfectamente la zozobra social que genera su diagnóstico.

-Es que fue otro de los gigantes abatidos. Resulta que el gran sueño de la libertad sexual acabó en condena de muerte por obra y gracia de esta enfermedad. A partir de ahí, Dios existe, y es como el que salía en Los diez mandamientos: un juez implacable, que no es ninguna broma ni abstracción.» 
personnages - Mauricio, La Porritos, Clotilde et leurs comparses -, et, d'autre part, comment il finit également par définir l'espace urbain où ils évoluent.

\section{Le desencanto des personnages}

À travers le personnage de Mauricio, dentiste aux idéaux toujours vivaces mais dépourvu de caractère, le romancier décrit un pays et ses habitants. Mauricio ne fait partie ni des puissants ni des lâches, personnages récurrents de l'univers des romans de Mendoza. Sa passion est son métier, l'odontologie, cependant il se sent en dette envers la société, c'est pour cela qu'il s'engage dans cette campagne électorale et finit par prendre goût aux meetings. Mendoza précisera dans l'entretien cité précédemment:

Le gusta su trabajo, pero siente que ello no basta para presentarse ante la historia. Pertenece a una generación con una responsabilidad histórica, pero no consigue justificarse a sí mismo, a pesar de que realiza un trabajo útil por el que además le pagan bien. Éste es el problema.

Il lui oppose des arrivistes - Fontán et les deux représentants du Parti Socialiste Catalan (Alemany et Fitó) - ou des laissés-pour-compte aux personnalités assez attachantes: les deux atrabilaires Brihuergas, vieux militant dévoué à la cause, et Serapio, prêtre-ouvrier qui aime la dive bouteille. À leurs côtés, se trouve La Porritos, chanteuse débordante de vie qui délaissera le combat politique pour son épanouissement personnel.

Mauricio est donc contacté par les amis de Fontán, qui dressent un bilan sans complaisance de la politique d'alors, lui expliquent les raisons pour lesquelles, lui Mauricio, devrait s'impliquer en politique, en Catalogne en particulier parce que la société civile doit être représentée (p. 25-26). Dans cette conversation, le mot reto, maître mot de l'époque, est prononcé comme le fera également Raurell, autre homme politique du PSC qui en a assez des clichés: tout comme le desencanto, le triomphalisme ne conduit nulle part et il reconnaît que le PSOE en gagnant les élections de 1982 a su relever le défi:

Al ganar las elecciones en el 82, el partido se enfrentó a este reto: adaptar una maquinaria económica y social obsoleta a la realidad interior y exterior. No podíamos seguir viviendo en una burbuja. (p. 38).

Au cours de ces différentes conversations, il apparaît que le mot democracia ne signifie pas la même chose pour tous et qu'à l'aube de ces élections autonomiques, le vote sanction risque bien dêtre choisi par les électeurs. Raurell invite donc Mauricio à accepter de se présenter sur les listes du PSC car l'Espagne, et en l'occurrence la Catalogne, connaît une nouvelle crise. Accepter, affirmera Raurell, signifie affronter un énorme travail de reconstruction après une longue période d'érosion. Ce n'est pas faire de la politique-spectacle, mais il est nécessaire de se rendre, par exemple, dans les associations de quartier, où se trouve le véritable électorat, le véritable destinataire de la politique gouvernementale (p. 41). Clotilde, la future compagne de Mauricio, lui déconseille fortement de 
se présenter - elle analyse clairement ces futures élections et sait que Jordi Pujol remportera les élections parce qu'il a parfaitement compris comment fonctionne le système: un negocio.

Mauricio franchit le pas, entre en politique. Il en fera les frais et comprendra cette crise de confiance qu'éprouve l'électorat : la politique n'est que compromission, perte des idéaux, enrichissement personnel pour les nantis, pauvreté pour les autres (p. 229).

Ces différents personnages réapparaissent soit au cours de la noce de Fontán («Una fantochada»), soit pour accompagner la dépouille de La Porritos à sa dernière demeure. Entre-temps, chacun s'est replié sur sa sphère privée et comme l'écrit un Mendoza assez moraliste, une ambiance désabusée trône dans la ville. Des individus sans scrupule y œuvrent à la faveur de l'ombre, et les finances y sont le nouveau moteur ${ }^{3}$. Ceux qui furent des étudiants en révolte se sont rangés (p. 219), la solidarité ne paraît plus de mise, les plus purs et les plus idéalistes semblent condamnés à mourir jeunes, voilà le constat amer que fait l'écrivain quand il explore avec une ironie mordante les diverses couches de la société.

Le regard corrosif que porte Mendoza sur le couple Mauricio-Clotilde plein de contradictions est assez comique. Qu'espère Mauricio, lui qui n'est pas un champion de la politique? Sa fatigue, ses désillusions se font plus pressantes et au cours du repas de noces de Fontán, il éprouve de la sympathie pour une adolescente inhibée qui ose intervenir dans la conversation autour des bienfaits supposés de la Transition. Sa repartie intempestive amuse Mauricio:

Por fin hay alguien que no nos toma en serio, pensaba Mauricio. Una nueva generación que juzga nuestros errores y se burla de nuestros sueños. Pronto se harán con el poder, impondrán sus ideas y sus fantasías y nos enviarán al asilo. Ellos, a su vez, fracasarán como hemos fracasado nosotros, pero al menos podremos descansar. (p. 293)

Cette cérémonie, à laquelle il prend part par convention, lui renvoie avec acuité son mal-être dans une société où seul le compromis assumé d'un commun accord permet aux gens de fonctionner au milieu de la confusion générale, sans se laisser écraser par l'absurdité et la contradiction. Mendoza décrit comment la vie sociale repose sur le cérémonial, le déguisement et le simulacre, éléments qui permettent à l'individu de survivre, de faire face à ses obligations pourvu qu'il croie en quelque chose. Ironiquement il ajoute que la foi fait bouger les

3 «Había un estado general de queja. El desenfreno de la época anterior había dejado paso a una red de relaciones mecánicas, un poco sucias. [...] En este ambiente medraban los sinvergüenzas y los que actuaban al amparo de las sombras. [...] La opinión pública se resignaba. Temas nuevos ocupaban la atención de la gente, temas triviales, que los medios de comunicación se encargaban de propagar y alimentar. [...] Los intelectuales cerraban la boca por temor a las represalias o por ambición, vendían su silencio e incluso su complicidad a cambio de dinero o de una fama pasajera y provinciana. La adulación y el cohecho volvían a abrir las puertas. Los políticos habían aprendido las posibilidades del poder y se injerían en todos los campos sin disimulo. [...] Las finanzas lo dominaban todo.» (p. 229-230). 
montagnes et toutes sortes de terrains, spécialement ceux qui permettent la spéculation immobilière (p. 305).

Que reste-t-il à Mauricio? Lamour qu'il ressent pour Clotilde? Mais même dans ce domaine, Mendoza chamboule le train-train quotidien qui pourrait s'installer. Crise du couple? Nous sommes dans les années 1980 et les relations homme-femme ont changé. Si Mauricio est très attiré par Clotilde, il avance pas à pas car cette jeune fille libérée le décontenance plus d'une fois. Il n'ose pas lui avouer tout simplement qu'il a envie de dîner avec elle car il ne peut prévoir les réactions de cette jeune fille moderne, il se protège donc en maniant l'ironie: «[...] pero tampoco quiero estamparte contra una roca». (p. 220). Quelle solution adopter? Avec quelque scrupule, il engage toutefois une relation parallèle avec La Porritos, chanteuse rencontrée lors de ses meetings politiques. Il est tiraillé entre deux femmes, ne sait que faire, écouter la raison, préférer le marivaudage. Il semble endosser la déprime d'une société déchirée. Avec la mort de La Porritos du SIDA, disparition hautement symbolique, s'achèvent les aspirations de Mauricio d'occuper une position non conventionnelle et son incursion dans un monde qui n'est pas le sien, mais s'achève aussi une époque où meurent les utopies, les illusions, et où l'idéal solidaire fait place au désir égoïste de prospérité. Débarrassé de son engagement politique embryonnaire, il se tourne alors vers Clotilde, plus conforme sociologiquement au modèle de celle qui devra être la femme de sa vie.

Qui est Clotilde? Mendoza montre la complexité de ce personnage déroutant, surtout pour Mauricio. Avocate stagiaire, elle apprécie Mauricio mais ne veut pas qu'on lui dicte sa conduite, pas plus qu'elle ne veut renoncer à son avenir. Pour exister professionnellement, elle accepte une mission pas très régulière à Genève, découvre le luxe, la finance internationale. On pourrait se demander pourquoi cette jeune fille très à gauche («muy politizada») accepte de compromettre ses idéaux? Tout simplement parce qu’elle veut prouver ses compétences, qu’elle s'ennuie mortellement dans les activités subalternes que le cabinet lui a proposées jusqu’à présent (p. 50): elle est décrite comme «precaria, supernumeraria». Elle se laisse contaminer par cette ambiance malsaine qui ne trouve de salut que dans l'argent. Ainsi enterre-t-elle ses rêves révolutionnaires, ses idéaux, ceux de la génération qui n’a pas connu la Guerre civile et qui espérait des jours meilleurs avec la Transition: "La alternativa era viajar en primera vestida de Armani y alojarse en Le Richelieu. Clotilde estaba confusa. Allí parecían acabar los sueños de su generación.» (p. 244).

Cette femme, désireuse de trouver une place dans la société par son travail, met en exergue la difficulté qu’ont les femmes dans ce domaine. La récurrence du mot «trabajo» dans sa diatribe souligne l'importance de cet acte qui devrait être un facteur d'équilibre individuel et d'épanouissement. Mendoza ne nous la rend pas très sympathique, par rapport à La Porritos qui aime Mauricio sans se poser de question. Par son comportement, Clotilde dévoile sa fragilité et toutes les contradictions d'une génération un peu puérile qui n’a pas réussi à réaliser son rêve de liberté et de justice et qui ne peut ressentir que du desencanto. 
Dans ce roman, à travers des dialogues légers, Mendoza confronte le lecteur à une réalité politique et sociale, à la fin d'une utopie. Tout comme la langue relâchée - citons pour exemple ce que dit Ruby, le frère de La Porritos: «Es ahora cuando he de estar aquí, al lado de mi pobre hermana, que la hago mucho falta. Si me voy, la doy un disgustazo que igual la acelera el final» (p. 328). Les jeunes manient volontiers le trait d'esprit, la blague, l'humour léger mais redoutable. Clotilde, qui se demande si elle est "bollera», n’hésite pas à employer des mots comme «follar» même quand elle s'adresse à son patron, un avocat de renom. Mauricio peut citer Goethe et dire: «vamos yendo» ou «coñazo». Mais les plus âgés, il est vrai, ne sont pas en reste: le père de Fontán loue la Bible qu’il lit tous les jours mais peut "envoyer paître " ses médecins: «a la porra».

Mendoza s'amuse à briser les stéréotypes parce que le relâchement de la langue est à l'image de cette nouvelle société en crise qui a perdu les repères traditionnels. Les éléments structurants que représentent les parents volent en éclat: la mère de Mauricio est séparée de son père, elle vit à Londres et semble être une adolescente attardée. La mère de Clotilde est une femme frustrée qui passe son temps à compenser ses manques en achetant sans cesse, en comparant les prix dans l'espoir de faire une bonne affaire, et son mari est un homme ennuyeux (p. 166). Enfin, pour La Porritos, issue d'une famille très pauvre qui se consacre à la contrebande de cigarettes à Tanger, tous les hommes font de la prison et toutes les femmes ont une vie dissolue. (p. 144).

Lauteur réussit le pari d'écrire un roman surprenant, «une comédie légère» ( «una comedia ligera») selon l'expression employée dans son précédent roman, une comédie douce-amère, dirons-nous, une comédie sentimentale doublée d'une étude de mœurs. Le lecteur assidu de Mendoza sera sans nul doute quelque peu dérouté par ces personnages ${ }^{4}$ mais il retrouvera avec bonheur son talent de conteur quand il offre à Barcelone, sa ville, un rôle prépondérant dans la construction du récit.

\section{Barcelone, à l'aube des dernières transformations}

Tout d'abord décor diffus, la ville passe progressivement au premier plan. À travers cette croustillante comédie sentimentale, Mendoza fustige les illusions perdues d'une époque qui est passée de l'utopie au business. Une nouvelle fois il

4 «La elección de una prosa deliberadamente llana no quita que a veces dé la impresión de mengua o pobreza (iy cuesta decir esto de un prosista magnífico como Mendoza!), como tampoco era indispensable una dispositio lineal como la que ha preferido. En la figura del protagonista absoluto, Mauricio, un idealista de tomo y lomo, en su solitaria profesión de odontólogo (tan solitaria como la de escritor) y en su sentido de la solidaridad y aun de la caridad, hay no poco de modelo contra el que se miden la estaturas de los cucañeros y sabandijas con los que se cruza, pero al cabo no deja de ser un personaje algo deslavazado que da la impresión de carecer de complejidad y que constituye un pedestal demasiado frágil para soportar el peso de toda la novela». (Ródenas de Moya 2007). 
chante la magie de sa ville. Il est amoureux de Barcelone, cette ville qui devient à la fois scène du récit conté et reflet de l'Histoire. Comme les personnages qui y évoluent, cette ville fétiche endosse la déprime d'une société déchirée entre desencanto et indifférence.

Et pourtant il suffirait d'un empujón dira Rubén, le cousin de Fontán, pour que Barcelone revienne au premier plan en parcourant tous les lieux touristiques bien connus. Il y perçoit les premiers frémissements liés à la candidature de la ville pour l'organisation d'une manifestation sportive. Ainsi le candidat Raurell expose à Mauricio le projet politique lié à l'organisation des Jeux Olympiques. Afin d'optimiser ce défi, il explique les modifications apportées à la ville dans le projet socialiste. Le point de départ serait la construction d'une rondaqui permettrait enfin la communication entre les différents quartiers. Il sait bien que certains protestent mais il balaie toutes ces oppositions au projet car elles sont sans fondement, Barcelone ne peut qu’en tirer bénéfice.

En détruisant la Barcelone crasseuse - elle sera soumise à la piqueta, dira Manuel Vázquez Montalbán dans son roman Sabotaje olímpico (Vázquez Montalbán 1993: 28) - la ville s'ouvrira différemment au monde mais aussi à la spéculation. Fitó le sait et il explique d’ailleurs que la productivité catalane si souvent louée n'est que le produit d'une adaptation réussie aux circonstances.

Barcelone change de physionomie au gré des visions contrastées de l'espace urbain que nous offre l'auteur. Il en dresse une sorte de plan en citant les noms de rues. Ce désir de vraisemblance, cette intention vériste permettent au lecteur d'identifier des lieux qui existent, mais qu'il ne s'y trompe pas, l'espace représenté dans le récit n'est pas, ou pas tout à fait, l'espace d'une géographie ou d'une topographie réelle ${ }^{5}$. Ces lieux offrent surtout à l'écrivain des espaces où faire évoluer des protagonistes emblématiques de leur milieu social, espaces qui soulignent le fossé qui s'agrandit entre les différentes couches sociales. Ces lieux sont le reflet du bouillonnement politique et sociétal que vivent l'Espagne et Barcelone en particulier. Le lecteur suit Mauricio dans ses meetings politiques. Ce représentant de la classe moyenne découvre un monde inconnu, le monde des banlieues - Ciudad Meridiana -: «Lo mismo podían estar en Barcelona que en Asia o en cualquier ciudad de América latina» (p. 69-70). Flanqué de Brihuegas, il parcourt un quartier similaire pour y rencontrer les habitants; ici, les maisons basses, aux murs blancs, sales et décrépis trahissent la pauvreté du lieu. Fréquentant La Porritos, il est amené à côtoyer la marginalité dans le quartier de Santa Coloma de Gramamet. Elle lui raconte sa vie, lui explique

5 (Francastel 1967, cité par Miterrand 1994:51-52). Ce qu'il écrit à propos de la peinture s'applique exactement à la littérature: "Distincte de l'objet et du motif, l'image figurative est le produit calculé, voulu, d'une organisation, matérielle et intellectuelle à la fois, du champ figuratif [...]. Espace perçu, espace pensé, espace fixé, espace de nouveau perçu puis interprété, des chaînes de relations s'établissent à partir des signes posés sur le support [...]. La saisie du figuratif ne peut être assimilée à la vue immédiate du monde naturel, puisqu'il s'agit du montage d'éléments intégrés à tous les niveaux, dans des structures intellectuelles complexes.» 
pourquoi elle a choisi de vivre à Barcelone, ville qui peut lui offrir l'opportunité de faire reconnaître ses talents artistiques (p. 145). Pour l'heure, elle se trouve au chômage. Comme beaucoup d'Espagnols venus d'ailleurs, Barcelone et sa région attirent mais noffrent pas de travail. «En Cataluña hay una crisis de mil demonios» dira Mauricio à Ruby; ce dernier, désireux lui aussi de trouver un emploi qui lui éviterait de retourner à Málaga, lui rétorque: "Pero Andalucía es una cosa, y Cataluña, ya se sabe» (p. 328) comme ébloui par le rayonnement illusoire des lieux. Mauricio enjolive ce qu'il voit. Il est sur une autre planète, comme dans les films de science-fiction de son adolescence (p. 155), mais La Porritos est là pour lui raconter ce qui s'y passe réellement: violence, drogue, prostitution, etc. (p. 199). Les habitants, pauvres voire misérables, semblent abandonnés à leur triste sort.

À ces quartiers dégradés, Mendoza oppose la Barcelone des classes aisées: l'oncle de Clotilde, qui l'a recommandée pour obtenir son emploi précaire, habite dans la rue Muntaner, quartier chic mais qui va à vau-l'eau à cause de la drogue, qui sévit partout (p. 123). Fontán réside dans un immeuble neuf - avec gardien en uniforme! - de la rue Anglí. Lappartement, spacieux, à la décoration recherchée, est un pur produit de décorateur professionnel. Clotilde, qui se rend chez Michelle, la fiancée de Fontán, non loin de là, sur le paseo de la Bonanova, est subjuguée par le triplex des parents, de haut standing. Un véritable "mausolée» selon les dires de leur fille (p. 276). Ces appartements au design recherché sont le reflet de cette nouvelle classe sociale branchée, qui se positionne comme étant à l'avant-garde, symbole d'une consommation urbaine qui dépasse les frontières de Barcelone.

Comme dans la présentation des quartiers, Mendoza s'attache à décrire les lieux où travaillent les protagonistes car ils contribuent de même à leur identification sociale. Le cabinet d'avocats où se trouve Clotilde est situé sur la Gran Vía, près de l'Hôtel Ritz, dans un appartement ancien et spacieux (p. 50). Le détective privé Cervello possède un bureau sale et désordonné, au troisième étage d'un immeuble ancien de la Ronda de San Antonio, qui correspond à son personnage de jorobeta matois capable de se montrer expéditif. Cervello tient des fiches sur toutes les personnes qui sont en relation de près ou de loin avec le cabinet d'avocats qui l'emploie: «No conviene dejar cabos sueltos. Si luego no hace falta, tanto mejor.» (p. 344).

Après son échec aux élections, - la défense des "petites gens » ne lui ayant pas réussi -, devenir l’associé de Manolo et s'installer à Mataró est une proposition alléchante. Au cours de l'achat de son cabinet dentaire, Mauricio va subir les mécanismes économiques qui font fonctionner cette nouvelle société. En effet, malgré sa position sociale, les banques lui refusent sa demande de prêtcar les garanties sont insuffisantes: "Ahora mandan los socialistas, sálvese quién pueda, usted ya me entiende» (p. 115). Felipe González ne fait pas l'unanimité, la prudence est le maître mot dans les milieux de la finance! En acceptant l'offre de prêt à un taux usuraire des amis de Fontán, il se frotte au monde capitaliste, lucratif, où les sentiments ne sont pas de mise. Mais Mendoza veut encore 
«sauver» son personnage en lui accordant une certaine dignité: Mauricio désire lui aussi gagner de l'argent, beaucoup d'argent mais pas dans una cochambre (p. 114).

Dans cette géographie de Barcelone qui se dessine et qui permet au lecteur d'associer lieux et personnages, Mendoza ne manque pas de faire allusion à l'un des parages privilégiés pour les Espagnols de tous bords. En effet, s'il est convenu de dire que la vie sociale se déroule d'ordinaire à l'extérieur, ce roman montre l'importance du bar ou du restaurant où se tiennent toutes sortes de discussions, où la vie privée n'est pas très bien préservée, selon les dires de Clotilde (p. 126). Tous les agissements importants des personnages ont lieu soit dans un bar soit dans un restaurant: le Bar Claret, point de chute de Mauricio et Clotilde; le restaurant italien Giardinetto témoin des échanges de certains hommes politiques sur la future Barcelone olympique, etc.

Mendoza fait référence avec précision à ces lieux de vie sociale car l'espace ainsi nommé souligne les changements de cette Barcelone où il est de bon ton d'adopter les nouvelles habitudes modernes. Barcelone est le reflet de cette société des années quatre-vingt où domine la figure de l'ejecutivo, tel Fontán et ses acolytes. Dans un entretien (La Vanguardia 02/04/2006), il répondra à la question sur son projet de "cartographier littérairement» les différentes phases historiques de Barcelone:

Aquí he contado la transformación de un grupo social en un momento en el que la evolución de la sociedad local era muy notaria. París, por aquel entonces, no cambió lo que Barcelona, Berlín, sí.

Le paraître semble donc de mise, les biens de consommation variés et divers deviennent indispensables, en particulier la voiture qui vous situe dans la société. Clotilde néchappe pas à cette nouvelle culture du bien de consommation. Posséder une voiture est son rêve, presque une obsession car, comme le rappelle Mendoza, dans cette ville, pour les plus déshérités, telle La Porritos, les déplacements se font en métro ou en bus. Mauricio, amoureux de sa Citroën trois portes, d'occasion, comprend qu'il ne peut pas lutter à armes égales avec Fontán et sa BMW. Ainsi saisit-il l'opportunité, quand le moteur rend lâme, de s'acheter une belle décapotable. Il va chercher La Porritos à l'hôpital: "Vaya, el famoso coche», s'exclame-t-elle affaiblie, et la raccompagne chez elle à Santa Coloma. Cette voiture assoit davantage sa notoriété parmi les plus humbles, les inégalités sociales se font plus criantes. Pris dans un embouteillage, Mauricio et Clotilde apprennent, déçus, que Barcelone organisera les Jeux Olympiques. C'est l'occasion, à la manière d'un vaudeville, de demander la main de Clotilde, de la surprendre afin d'obtenir son accord. Cet embouteillage, cette demande en mariage sont tout un symbole. À leur manière, c'est comme s'ils délaissaient le passé - ils viennent d'enterrer La Porritos - et leurs idéaux et se tournaient vers un nouvel avenir, entraînés eux aussi par cette douce euphorie. L'enthousiasme de la foule vire à la folie, quand elle apprend les résultats du CIO. Mauricio et Clotilde en sont surpris, mais à ce moment ils ne se doutent pas encore que 
l'événement va transformer ce port industriel de la Méditerranée, cosmopolite et multiculturel, en une des villes les plus glamour d'Europe.

Par le choix de ces personnages évoluant dans un espace et une époque ancrés dans la fameuse période de Transition, Mendoza s'ingénie à mêler vérité fictionnelle et vérité historique et permet ainsi à son lecteur de mieux appréhender la complexité d'une époque clé de l'Histoire récente.

Ville, personnages, destin historique et social s'entrecroisent, proposant un bilan moral et idéologique d'une époque et d'un pays où les gens sont conscients qu'ils vivent la fin d'une utopie, la fin d'une époque de géants politiques (p. 365). Mendoza nous offre une vision polyphonique de nature fictionnelle de cette période délicate mais ne veut-il pas nous montrer aussi que ces elecciones primarias sont les choix que font les personnages dans leur vie quotidienne, choix qui ne sont finalement pas multiples et variés?

\section{Bibliographie}

Francastel, P., 1967, La Figure et le lieu, t. III, Paris, Denoël.

Fancelli, A., Mendoza, E., 26/03/2006., «El escritor de la ciudad prodigiosa», Madrid, El País semanal.

Miterrand, H, 1994, L'illusion réaliste, Paris, PUF.

Mendoza, E., 1996, Una comedia ligera, Barcelona, Seix Barral.

Mendoza, E., 2006, Mauricio o las elecciones primarias, Barcelona, Seix Barral.

Moix, L., Mendoza, E., 02/04/2006., "Entrevista, Barcelona», La Vanguardia.

Ródenas de Moya, D., abril 2007, «La narrativa española de $2006 »$, Insula, nº 724.

Vázquez Montalbán, M., 1993, Sabotaje olímpico, Barcelona, Planeta. 